\title{
Effects of thermal aging on the cyclic thermal shock behavior of oxide/ oxide ceramic matrix composites
}

\author{
Zhengmao Yang ${ }^{\mathrm{a}, *}$, Hui Liu ${ }^{\mathrm{b}}$ \\ ${ }^{a}$ Institute of Mechanics, Chinese Academy of Sciences, Beijing, China \\ ${ }^{\mathrm{b}}$ School of Aerospace Engineering, Tsinghua University, Beijing, China
}

\section{A R T I C L E I N F O}

\section{Keywords:}

Ceramic matrix composites

Cyclic thermal shocks

Thermal aging

Hierarchical porosity

Strain energy release rate

\begin{abstract}
A B S T R A C T S
A particular emphasis is placed on the thermal aged oxide/oxide ceramic matrix composites under cyclic thermal shocks, which exhibit hierarchical internal structures spanning multiple volume/scales. The present work focuses on establishing thermo-mechanical loading conditions-structure-property linkages for the thermal aged oxide/ oxide ceramic matrix composites under cyclic thermal shocks. Firstly, the matrix micro-cracks and delamination were identified by SEM observation, and the compressive mechanical behaviors were studied after different cyclic thermal shock numbers. The thermal aging process resulted in apparent changes in thermal shock resistance of materials. Considering the material deterioration induced by the thermal aging process, aging-related factor was introduced to predict the compress behaviors of the thermal aged oxide/oxide ceramic matrix composites under cyclic thermal shocks. The correlation between porosity and strain energy release rate was also constructed, which provided an excellent agreement with the experiments.
\end{abstract}

\section{Introduction}

Ceramic matrix composites (CMCs) exhibit outstanding properties of high strength, high stiffness and stiffness-to-density ratios as well as good thermal stability at high temperature [1,2], which have aroused the interest of many researchers to consider them as the attractive candidates for high-temperature applications, such as aerospace propulsion system components and thermal protection systems for re-entry vehicles $[3,4]$. The structural materials for aero-engine applications require the long-term thermal durability under a variety of conditions involving temperature and loadings, not only in constant states but also under cyclic thermal shocks. More specifically, two typical types of thermo-mechanical damage would be encountered when the composite structures service with high temperature combustion environment: thermal cycling especially cyclic thermal shocks (thermal fatigue) and long exposure to elevated temperatures (aging), which can cause changes in the morphology, strength and stiffness [5,6]. Unfortunately, the knowledge on the mechanical behavior of thermal aged oxide/oxide CMCs under cyclic thermal shocks is rather limited. Consequently, it is necessary to investigate the effect of thermal aging on the cyclic thermal shocks behavior for theses composites.

The long-term thermal aged CMCs have been thoroughly investigated. Xu et al. [7] observed the tensile strength of $\alpha$-alumina Nextel 610 fibers under exposure temperatures of $900^{\circ} \mathrm{C}, 1100^{\circ} \mathrm{C}$ and $1300{ }^{\circ} \mathrm{C}$, the results show that the grain growth and defects induced during high-temperature exposure contribute to the loss of the fiber strength. Carelli et al. [8] presented the changes in the mechanical properties of an all-oxide fiber-reinforced composite with long-term exposure $(1000 \mathrm{~h})$ at temperatures of $1000-1200^{\circ} \mathrm{C}$ in air, which suggests that the matrix and the fiber/matrix interfaces seem to be strengthened during aging, but the main tensile properties, including strength and failure strain in the $0^{\circ} / 90^{\circ}$ orientation, are unchanged. Kostopoulos et al. [9] investigated the anisotropic damage of alumina/alumina continuous fiber ceramic composites under a corrosive/high-temperature exposure, in which stiffness matrix components and strength were experimentally defined as a function of exposure duration. Cluzel et al. [10] developed a model for the mechanical behavior and lifetime of CMCs with self-healing matrix, the result shows that the coupling of mechanical and physicochemical mechanisms is important in predicting the behavior of materials in the complex thermo-mechanical and oxidizing environment. Vokmann et al. [11] studied the effects of heat treatments on the mechanical and microstructural properties of a fiber-reinforced weak interface composite in the oxidative atmosphere. CMCs with alumina, SiOC-based

\footnotetext{
* Corresponding author.

E-mail address: zmyang@imech.ac.cn (Z. Yang).
} 
Table 1

Mechanical and thermal properties of the fiber and matrix in original oxide/ oxide CMCs at room temperature [21,22].

\begin{tabular}{|c|c|c|c|}
\hline \multirow[t]{2}{*}{ Materials phases } & CTE & $E$ & $\sigma_{\mathrm{u}}$ \\
\hline & $\left(10^{-6} /{ }^{\circ} \mathrm{C}\right)$ & (GPa) & (MPa) \\
\hline $99 \% \alpha-\mathrm{Al}_{2} \mathrm{O}_{3}$ fiber & 8.8 & 373 & 2930 \\
\hline $85 \% \mathrm{Al}_{2} \mathrm{O}_{3}-15 \% 3$ YSZ matrix & 10.2 & 210 & $\approx 45$ \\
\hline
\end{tabular}

ceramics, and $\mathrm{ZrO}_{2}-\mathrm{Al}_{2} \mathrm{O}_{3}$ matrix were further investigated in Ref. [12] and the obtained results indicated the importance of microstructural investigations for CMCs. Hay et al. [13,14] presented the possible relationships between strength and grain-size and other causes of strength degradation after heat-treated at $1100^{\circ} \mathrm{C}-1500^{\circ} \mathrm{C}$ for $1 \mathrm{~h}-100 \mathrm{~h}$ in air. Almeida et al. [15] observed the behavior of an oxide fiber at elevated temperatures before and after thermal exposure. The heat treatments increase the fiber stability and creep resistance but reduce the tensile strength, which is related to the observed microstructural transformations.

Experimental research has been also conducted on the mechanical properties of oxide/oxide CMCs under cyclic thermal shocks [16-18]. Recently Yang et al. [19,20] investigated cyclic thermal shock-induced thermo-mechanical damage of oxide/oxide CMCs, they found that micro-cracks in the matrix lead to the macroscopic damage, which is induced by the thermal stress during the shocks. Due to its complex microstructure, the thermal aged CMCs are expected to possess complicated mechanical behavior under cyclic thermal shocks. However, much research has been devoted to the mechanical properties of CMCs after long-term aging as above, few studies have been published on the contribution of the long-term thermal aging to the cyclic thermal shock resistance of CMCs.

In the present work, the 2-D woven oxide/oxide CMCs with different thermal aging conditions (aging duration up to $500 \mathrm{~h}$ at a temperature of $1200^{\circ} \mathrm{C}$ ) were tested under cyclic thermal shocks. The microstructural evolution and mechanical behaviors of thermal aged CMCs under cyclic thermal shocks were analyzed in detail by the hierarchical porosity and compressive modulus. The aging-related factor from the thermal aging process was proposed and introduced to the compress modulus prediction. The correlation between porosity and strain energy release rate was also obtained, which can provide references for the hot end components design of a gas turbine.

\section{Materials and experiments}

\subsection{Materials description}

The 2-D woven oxide/oxide CMCs investigated in the present work consist of the $\mathrm{Al}_{2} \mathrm{O}_{3}-\mathrm{SiO}_{2}-\mathrm{ZrO}_{2}$ matrix and the reinforced fiber Nextel ${ }^{\mathrm{TM}}$ $610\left(99 \% \alpha-\mathrm{Al}_{2} \mathrm{O}_{3}\right)$ that exhibits advantageous specific mechanical properties including strength and stiffness. The uncoated Nextel ${ }^{\mathrm{TM}} 610$ fibers are in 8-harness satin weave (8HSW) with a volume fraction of approximately $44 \%$ and a density of $4.0 \mathrm{~g} / \mathrm{cm}^{3}$, with $0^{\circ} / 90^{\circ}$ woven layers. The matrix has the chemical compositions of $85 \mathrm{wt} \% \mathrm{Al}_{2} \mathrm{O}_{3}$ and $15 \mathrm{wt} \% 3 \mathrm{YSZ}\left(3 \mathrm{~mol} \%\right.$ Yttria-stabilized monoclinic and tetragonal $\mathrm{ZrO}_{2}$ ), the density of which is $4.2 \mathrm{~g} / \mathrm{cm}^{3}$. The CMCs are made by a vacuum bag process under low pressure and low temperature followed by a pressureless sintering technique.

The coefficient of thermal expansion (CTE), elastic modulus and ultimate tensile strength of the original oxide/oxide CMCs are listed in Table 1 . As the total density of the original CMCs is $2.71 \mathrm{~g} / \mathrm{cm}^{3}$, taking into account the density of fibers and matrix, the materials possess a total porosity of $34 \%$. Due to the high porosity, the effective property of materials must be much lower than those summarized in Table 1.

Fig. 1 shows the porous microstructure of the original CMCs. There are numerous shrinkage matrix cracks exist on the specimen surface, which are formed by the mismatch of CTE between matrix and fibers during processing [19]. The microstructure of the cutting surface in Fig. 1 (a) reveals that there are a lot of cylindrical pores in size of $>$ $100 \mu \mathrm{m}$ distributed in the fiber direction and the thickness. The pore surface is smooth and uniform, which indicates that the pores exist before cutting and may affect the material stiffness. As shown in Fig. 1 (b), the fibers can be considered to be dense compared with the matrix, and there are some micro-pores around the fibers.

Fig. 1(c) implies that the matrix is a collection of alumina particles and micro-pores, and the size of micro-pores is less than $50 \mathrm{~nm}$ which is much smaller than that of alumina particles. If the particles are approximated as ellipsoids, the porosity of micro-pores in matrix can be calculated as $48 \%$. As the matrix volume fraction is $56 \%$, the micropores in matrix account for $28 \%$ of the material volume, which means that there are ca. 7\% micro-pores in fiber plies [20].

\subsection{Experiments}

\subsubsection{Thermal aging tests}

Currently, a temperature of $1200^{\circ} \mathrm{C}$ is chosen for the thermal aging test of composites, which is approached the maximum recommended operating temperature of the Nextel ${ }^{\mathrm{TM}} 610$ fibers. As for the extreme environmental problems such as thermal overload, it is without the scope of the present work. To simulate the working conditions of materials in the gas turbine, the specimens were isothermally exposed to a high temperature environment of $1200^{\circ} \mathrm{C}$, and aged from $100 \mathrm{~h}$ to $500 \mathrm{~h}$.

\subsubsection{Cyclic thermal shock tests}

On completion of the thermal aging treatments, the cyclic thermal shock tests (thermal down shock) were performed for the specimens. Firstly, the specimens were put into a muffle furnace preheated to the desired temperature of $1100^{\circ} \mathrm{C}$ in accordance with the application of materials, held for $10 \mathrm{~min}$ for homogenization, then quenched in distilled water $\left(20^{\circ} \mathrm{C}\right.$ of bath temperature). When the specimens have cooled to the room temperature, they were dried and inspected for visible damage. The thermal shock process was repeated to study the effects of cyclic thermal shocks. More details about the thermal shock tests can refer to Ref. [19].
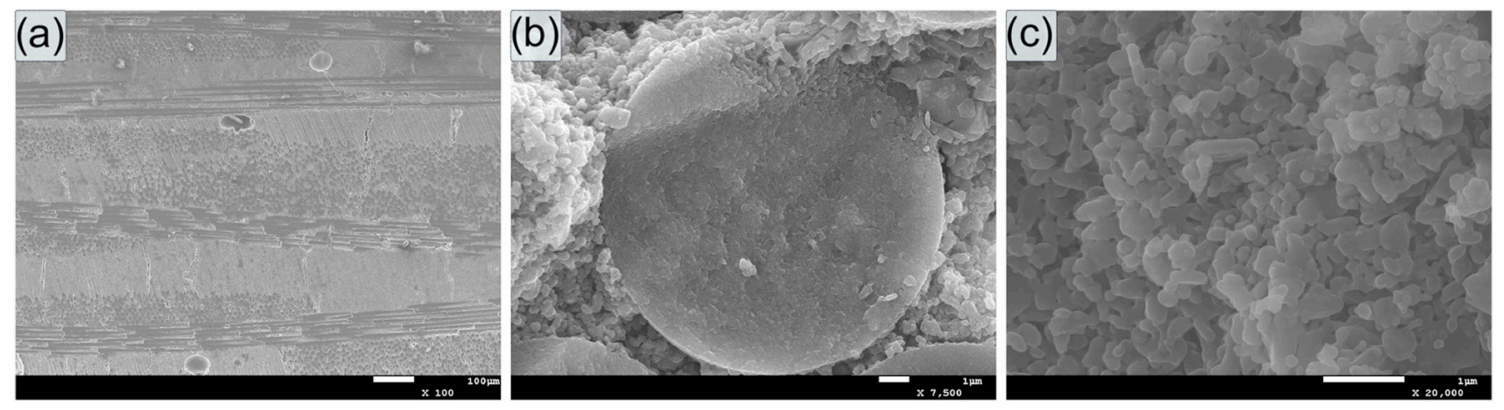

Fig. 1. Microstructure of the original 2-D woven oxide/oxide CMCs. (a) Overview, (b) fiber and matrix, (c) the porous matrix. 
Table 2

Thermal aging and cyclic thermal shock conditions for all tested specimens.

\begin{tabular}{lllll}
\hline Material States & \multicolumn{4}{l}{ Cyclic thermal shocks $(N)$} \\
\hline Thermal aging duration $(h)$ & 0 & 1 & 4 & 8 \\
0 (original) & I & - & - & - \\
100 & II & - & V & VI \\
200 & III & - & VII & VIII \\
500 & IV & IX & X & XI \\
\hline
\end{tabular}

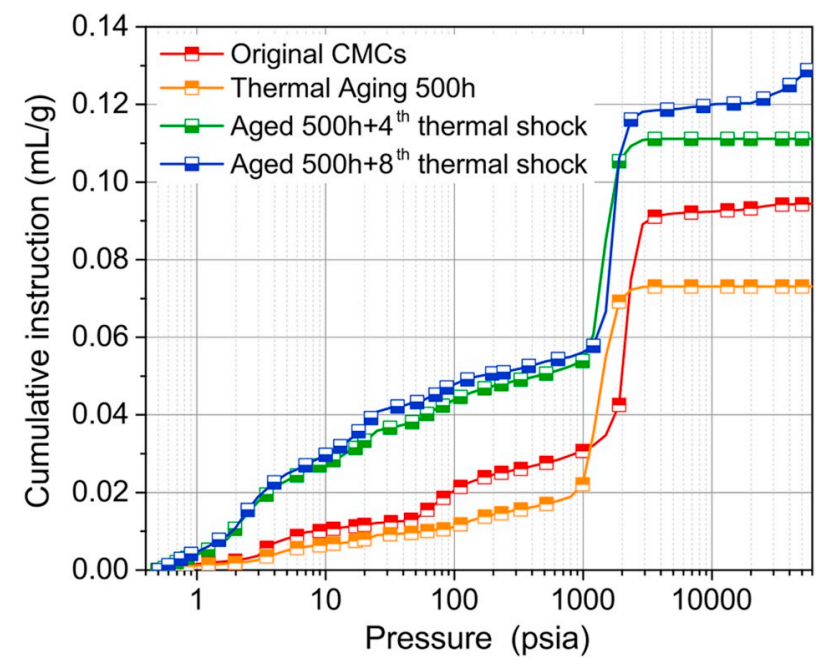

Fig. 2. The relationship between the applied pressure and the cumulative mercury intrusion.

The experimental protocol employed in this study is shown in Table 2, each state corresponds to a set of specimens. All the specimens were inspected before tests in order to ascertain their initial state as the reference state, which is a prerequisite for the subsequent evaluation of the test results.

\subsubsection{Mercury intrusion porosimetry tests}

The mercury intrusion method is widely used in measuring the porosity of porous materials. The relationship between the pressure applied to mercury and the radius of pores through which mercury can penetrate is as follows:

$\operatorname{Pr}=-2 \gamma \cos \theta$

where $P$ is the pressure applied to mercury; $r$ is the pore radius; $\gamma$ is the surface tension of mercury and $\theta$ is the contact angle between mercury and the test materials. The formula apparently provides a simple and convenient relationship between pressure and pore radius. As the pressure increases, the mercury will gradually enter the finer pores, the distribution of pore radius can then be determined by tracing the amount of mercury entering the pores and the increasing pressure provided.

To determine porosity as well as generate the pore size distribution of the thermal aged CMCs under cyclic thermal shocks, a Micromeritics Autopore IV 9510 porosimeter is used. After performing the predetermine thermal process, the samples were put in a sample tube of which the volume was measured, and the sample tube was vacuumed below $50 \mu \mathrm{m} \mathrm{Hg}$ through the vacuum pump in the system, then filled with mercury and pressurized to $0.001-0.003 \mathrm{MPa}$.

Fig. 2 reveals the pressure-cumulative mercury intrusion curve for four different states of CMCs: state I, state IV, state X and state XI, as listed in Table 2. The mercury entering the sample can be divided into two parts: one is the mercury entering the sample at lower pressure, and the mercury volume accounts for the large particle gap volume in the sample, which is presented as manufacturing micro defects, the other is the mercury entering the sample at higher pressure, which implies the volume of nano-voids in the particles.

\subsubsection{Mechanical test}

In order to quantify the effects of thermal shock cycles on the
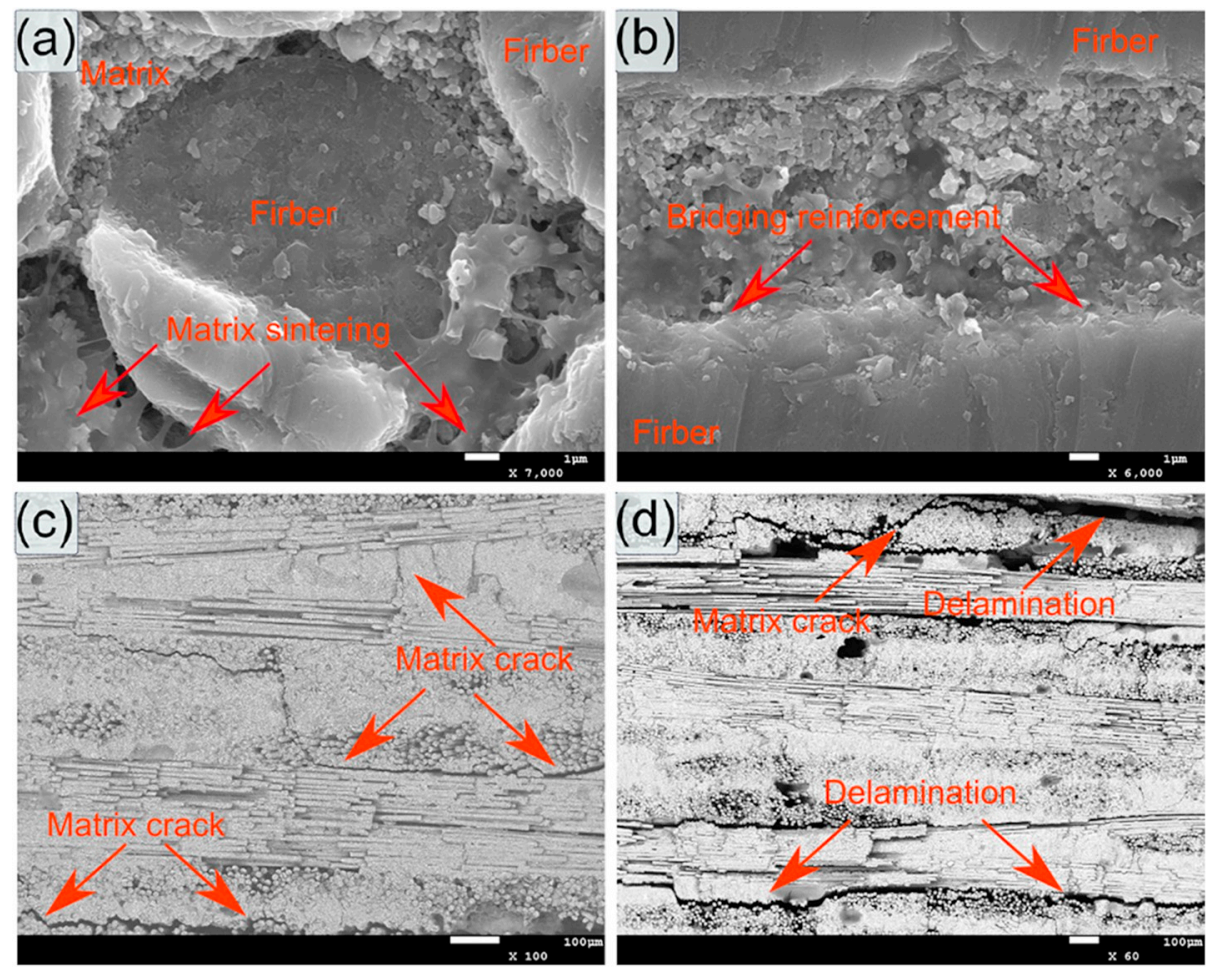

Fig. 3. Microstructure of the thermal aged oxide/oxide CMCs under cyclic thermal shocks. (a) Matrix sintering; (b) Fibers/matrix bridging; (c) Matrix cracks; (d) Delamination. 


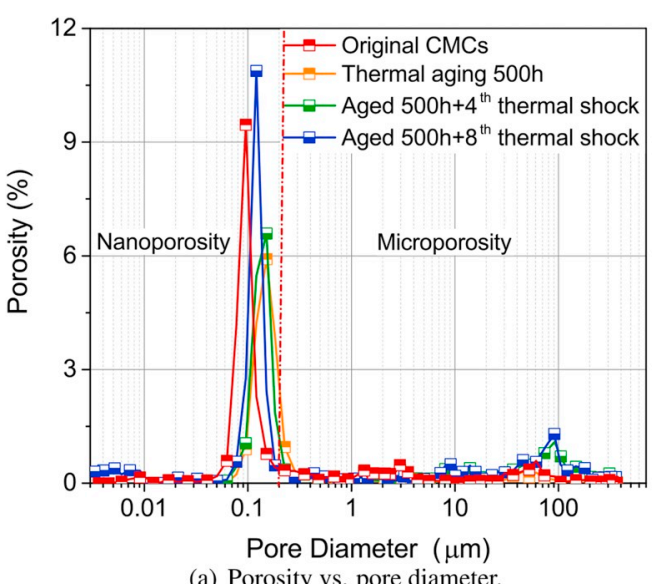

(a) Porosity vs. pore diameter.

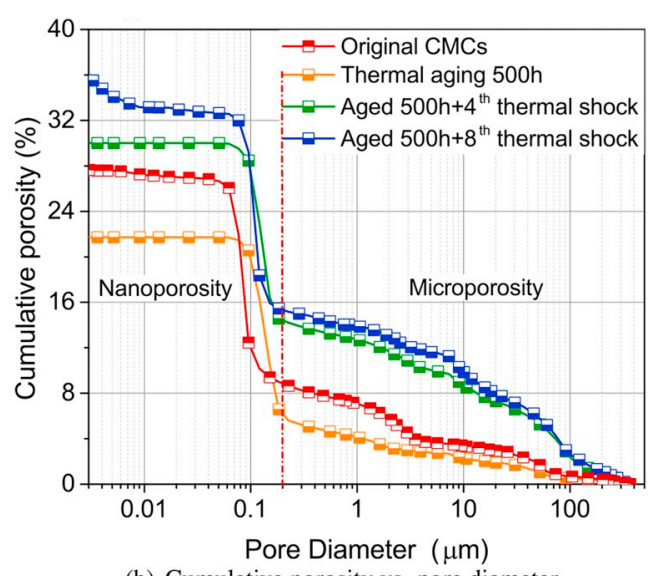

(b) Cumulative porosity vs. pore diameter.

Fig. 4. Pore size distributions determined by the mercury intrusion porosimetry.

mechanical properties of thermal aged CMCs, the in-plane mechanical properties of CMCs were investigated by uniaxial monotonic compressive loadings in the fiber direction (the weft direction). The compressive specimens were machined into the shape of $30 \mathrm{~mm} \times 20 \mathrm{~mm}$ using the water jet, and the gauge length is $15 \mathrm{~mm}$ [23]. All tests were performed by the servo-hydraulic MTS Bionix testing machine at room temperature, with a constant displacement rate of $0.06 \mathrm{~mm} \cdot \mathrm{min}^{-1}$.

\section{Microstructural characterization}

Due to the highly transient processes of thermal shock and the restrictions of the temporal and spatial resolution for current experimental methods, it is difficult to do the in-situ observations during thermal shock tests. Thus, the understanding of the mechanism of microstructure evolution for the thermal aged materials under cyclic thermal shocks is still limited.

However, thermal shock or aging mechanical responses are always related to their microstructure, such as the matrix cracks, fiber/matrix interface. As the pore scale in fiber and matrix of CMCs is inconsistent that the effect on material mechanical properties is different, the hierarchical porosity concept is thus proposed to describe the effect of porosity on the mechanical performance of porous matrix CMCs. The various pores observed can be broadly classified as: (i) Micro-pores refer to pores larger than $200 \mathrm{~nm}$ as regards to mercury intrusion, including matrix voids, intraply and interply micro-pores. (ii) Nano-pores are considered as pores smaller than $200 \mathrm{~nm}$, existing in matrix between the alumina-zirconia particles.

Therefore, in the current work, the scanning electron microscope (SEM), hierarchical porosity method and compress tests are used to explore the correlations between microstructure and mechanical behavior of thermal aged CMCs under cyclic thermal shocks.

\subsection{Microstructural evolution}

In order to study the internal microstructural evolution of thermal aged materials under cyclic thermal shocks, SEM was used to scan the specimens, as shown in Fig. 3. After $500 \mathrm{~h}$ of thermal aging, there are some distinct changes in the matrix and interface microstructure: (i) The sintering occurs between particles of the matrix and at the interface of the fiber and matrix, as shown in Fig. 3(a), which reduces the porosity in the matrix-rich region; (ii) The materials become compact, because of the partial closure of micro-cracks and the filling of pores caused by the high-temperature aging treatment; (iii) The bridging reinforcement occurs due to the sintering, as illustrated in Fig. 3(b).

The microscopic observation of the thermal aged $500 \mathrm{~h}$ specimens under 8 thermal shocks shows that there are two kinds of failure mechanisms that affect the overall material property differently, as illustrated in Fig. 3(c)-(d):

(i) Micro-cracks in the matrix distributed along with the thickness induced by the in-plane tensile stress. These micro-cracks are formed due to the low-toughness and high strength characteristics of the matrix, and the crack density in matrix increases with thermal shock cycles [20].

(ii) Delamination between the fibers and matrix within the boundary. This delamination is caused by the high temperature gradients and different CTE in fibers and matrix.

\subsection{Variations of the porosity}

As analyzed previously, the internal mechanism of micro-pore evolution is the increase of matrix micro-crack density, which may cause the failure of thermal aged CMCs under cyclic thermal shocks. Therefore, the matrix microstructure evolution of thermal aged CMCs under cyclic thermal shocks can be characterized by the increase of micro-pores porosity.

Fig. 4(a) illustrates the porosity and pore diameter distribution for the four different CMC states mentioned above. The maximum value (peak) is approximately reached at a pore diameter of $100 \mathrm{~nm}$ for all states, but for state IV, the peak shifts slightly to the right. However, as for state $\mathrm{X}$ and state $\mathrm{XI}$, the maximum pores gradually increase, and the peak position shifts slightly to the left, the value increases with the cumulative number of thermal shock cycles.

Fig. 4(b) shows the cumulative porosity distribution of materials in the four states. The porosity of thermal aged CMCs increases with the cumulative number of thermal shock cycles, and the nanoporosity takes the major part of the total porosity. In addition, the porosity of thermal aged CMCs is about $6 \%$ lower than that of the original materials, which is due to the partial closure of micro-cracks effectively distributed between particles of the matrix effectively.

To describe and quantify the microstructural evolution of thermal aged CMCs under cyclic thermal shocks, the change in microstructure can be characterized by the micro-porosity increment $\Delta \zeta$ uniformly. The variation of the porosity can be expressed by,

$\Delta \zeta=\zeta-\zeta_{0}$,

where $\zeta$ denotes the current porosity of both nano and micro pores, and $\zeta_{0}$ is the initial porosity of the original CMCs; $\Delta \zeta$ represents material microstructural evolution.

For the thermal aged $500 \mathrm{~h} \mathrm{CMCs,} \mathrm{Fig.} 5$ elucidates that the porosity increment caused by the cyclic thermal shocks is not started from 0 . The change in the porosity of thermal shocked CMCs is larger compared to original CMCs, indicating that the thermal shock resistance of 


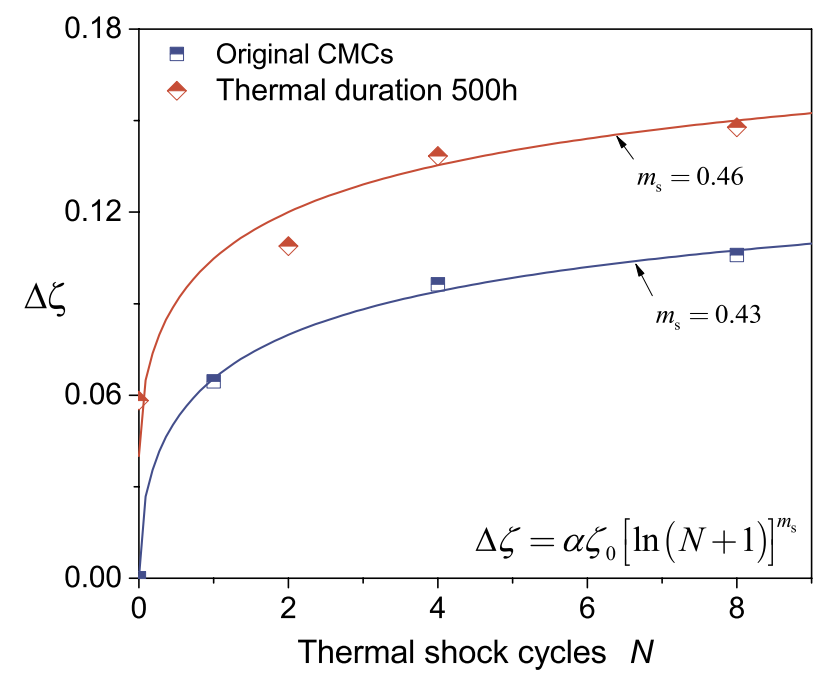

Fig. 5. Porosity increment $\Delta \zeta$ as a function of the thermal shock cycle number $N$ at shock temperature $1100^{\circ} \mathrm{C}$.

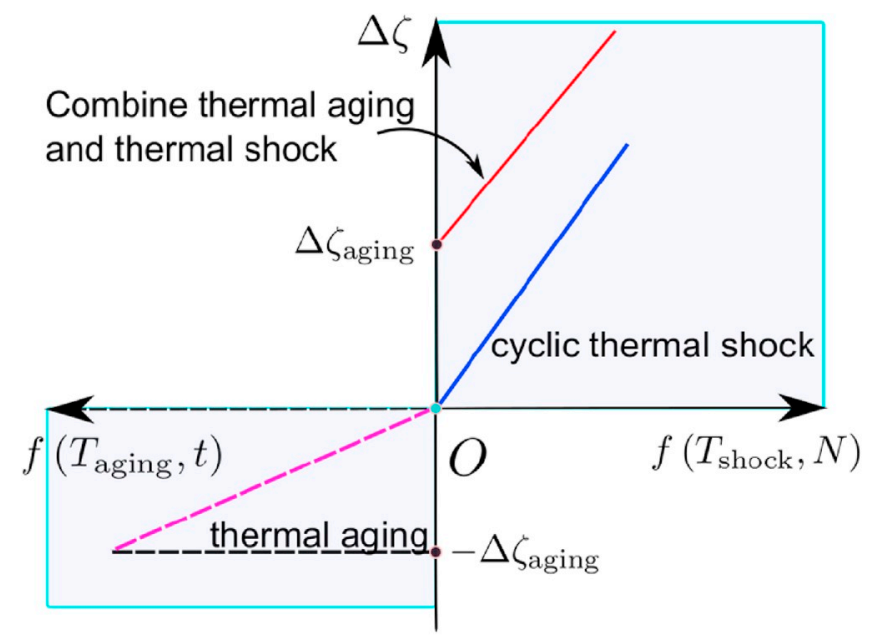

Fig. 6. Schematic of porosity increment in the thermal aged CMCs under cyclic thermal shocks.

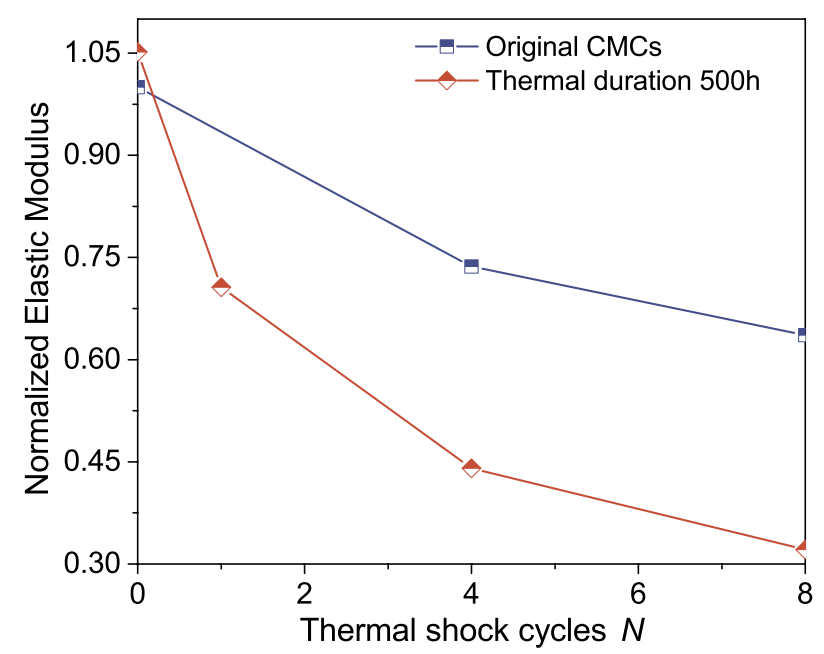

Fig. 7. Compressive mechanical properties as functions of the thermal shock cycles $N$.

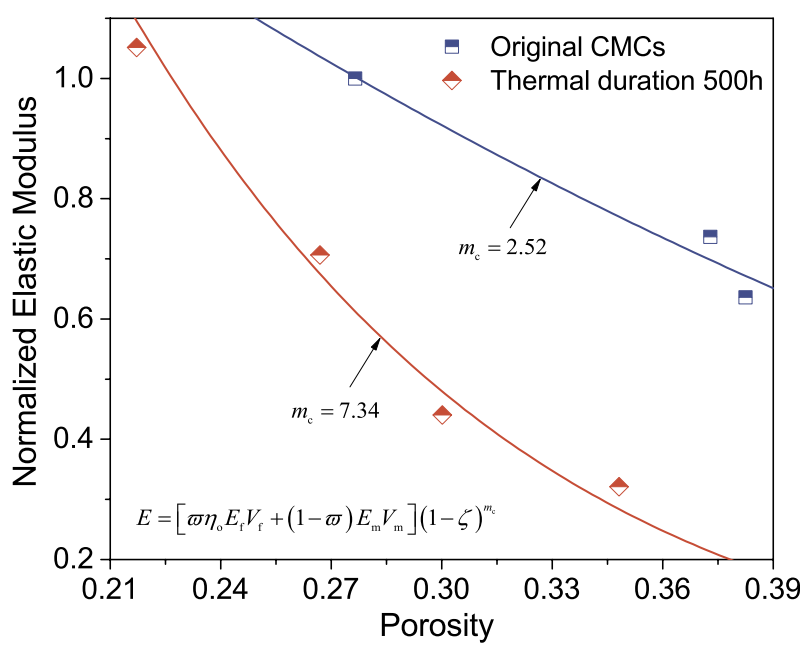

Fig. 8. Comparison between experimental measurements and predictions from the present models for the correlation between elastic modulus and porosity.

composites is weakened by the long-term thermal aging. Evolution of the different parts of porosity increment is illustrated in Fig. 6, the initial porosity increment value is precisely equal to the thermal aging-induced porosity.

The decrement of microporosity can be approximated by a logarithmic function of thermal shock cycle number $N$,

$\Delta \zeta=\alpha \zeta_{0}[\ln (N+1)]^{m_{s}}$

where $\alpha$ and $m_{\mathrm{s}}$ are the fitting parameters of the approximation. As shown in Fig. 5 , the shape parameter $m_{s}$ of the original CMCs is inconsistent with that of thermal aged $500 \mathrm{~h} \mathrm{CMCs,} \mathrm{indicating} \mathrm{that} \mathrm{there} \mathrm{is} \mathrm{a}$ coupling effect between cyclic thermal shocks and long-term thermal aging.

\subsection{Variations of the mechanical performance}

Fig. 7 reveals the correlation between the normalized compression modulus and thermal shock cycles $N$ of the original and thermal aged CMCs. The reduction trend for each thermal shock cycle $N$ is referring to a set of specimens, and compressive elastic modulus are normalized according to the initial values. As shown in Fig. 7, before performing thermal shocks, the thermal aging duration progresses lead to the increase of elastic modulus compared to the original CMCs, which may be due to the matrix densification and the continuously increasing fiber/ matrix interface strength. However, when subjected to the cyclic thermal shocks, the thermal aged CMCs show a rapid decrease in elastic modulus compared to the original CMCs, suggesting that the thermal aging treatment reduces the thermal shock resistance of materials.

The micromechanical models of composites can be obtained based on the properties of individual components and their arrangement [24]. Properties such as the elastic modulus and the relative volume fractions of both fibers and matrix are the fundamental quantities used to predict the properties of composites. The rule of mixture is a widely accepted micromechanical model for calculating the elastic modulus, as

$E_{\mathrm{ROM}}=E_{\mathrm{m}} V_{\mathrm{m}}+E_{\mathrm{f}} V_{\mathrm{f}}$

where $E_{\mathrm{ROM}}$ is the theoretical value of the composite elastic modulus obtained from the rule of mixture (ROM). $V_{\mathrm{f}}$ and $V_{\mathrm{m}}$ are the volume fractions of fiber and matrix in the composites, respectively, and $V_{\mathrm{f}}=$ $1-V_{\mathrm{m}}$. Eq. (4) implies that the elastic modulus is affected by the volume fractions of both fiber and matrix.

However, the variations of porosity in materials cannot be ignored. Hence, the modified ROM, considering the total porosity, can be written as: 


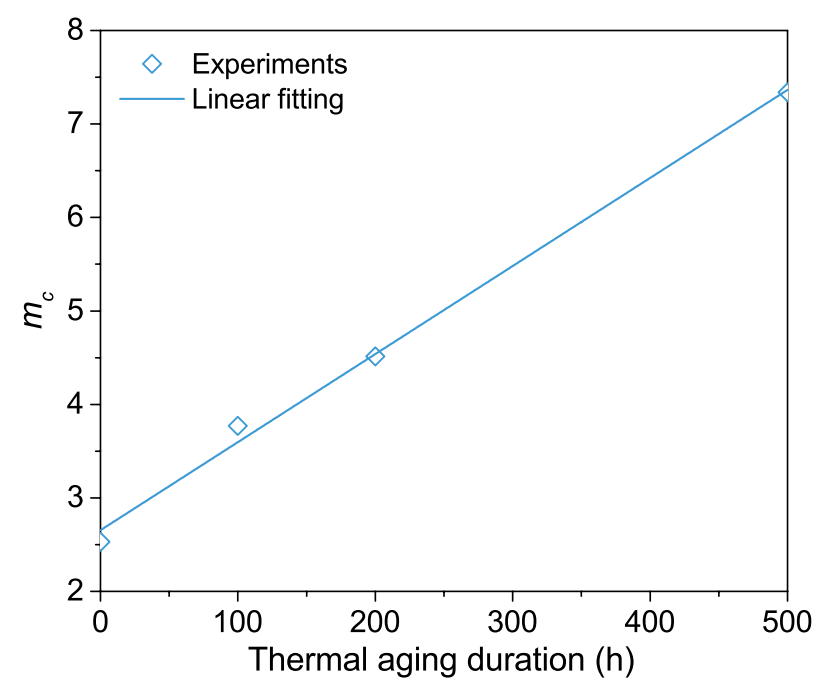

Fig. 9. The relationship between $m_{\mathrm{c}}$ and the thermal aging duration.

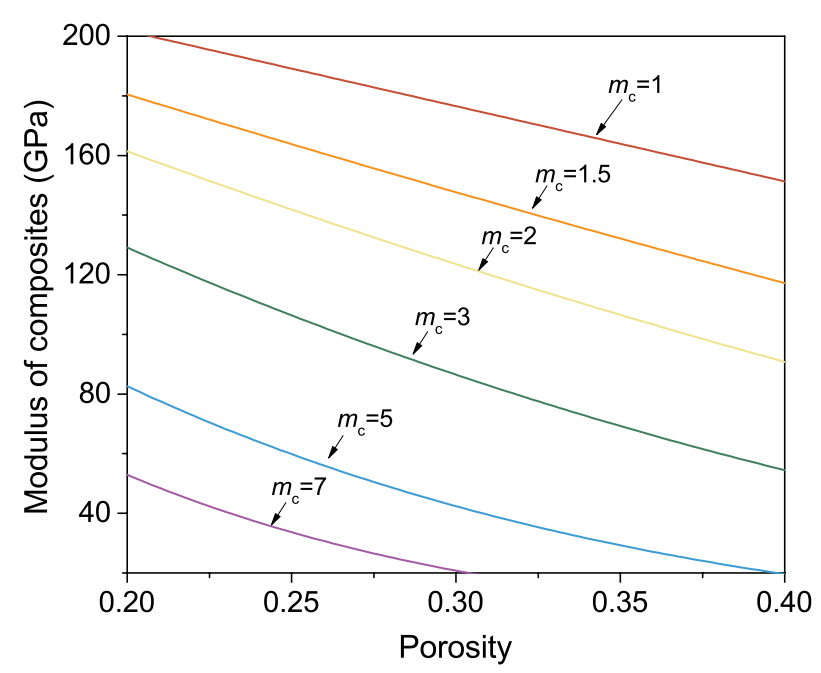

Fig. 10. Effect of $m_{\mathrm{c}}$ on prediction of effective modulus for porous matrix CMCs.

$E=\left[\varpi \eta_{\mathrm{o}} E_{\mathrm{f}} V_{\mathrm{f}}+(1-\varpi) E_{\mathrm{m}} V_{\mathrm{m}}\right](1-\zeta)^{m_{\mathrm{c}}}$,

where $\varpi$ is a weight factor representing the influence of fiber and matrix; $\zeta$ is the total porosity of materials; $\eta_{\mathrm{o}}$ shows the effects of the fiber orientation, as suggested in Ref. [25], here $\eta_{\mathrm{o}}=1$. Exponential $m_{\mathrm{c}}$ is a material parameter, containing the evolution of composite stiffness.

Fig. 8 shows the composite stiffness degeneration as a function of porosity for the original and thermal aged $500 \mathrm{~h}$ CMCs under cyclic thermal shocks. The results confirm that the model coincides well with the experiments. Interestingly, the factor $m_{\mathrm{c}}$ of thermal aged $500 \mathrm{~h} \mathrm{CMCs}$ is determined as 7.34, while $m_{\mathrm{c}}$ of original materials is only 2.52 , indicating that $m_{\mathrm{c}}$ rises after long-term thermal aging, and the material properties may change after thermal aging process. Since the aging temperature $T_{\text {aging }}$ is a fixed value, $m_{\mathrm{c}}$ is considered as a function of the long-term thermal aging duration $t$. It should be noted that $m_{\mathrm{c}}$ for the composites under tensile and compress loadings are slightly inconsistent, due to the various damage mechanism for CMCs under different loading cases, referred to Ref. [26].

To investigate the relationship between $m_{\mathrm{c}}$ and the thermal aging duration, the $m_{\mathrm{c}}$ for thermal duration $100 \mathrm{~h}$ and $200 \mathrm{~h}$ are obtained using Eq. (5). Fig. 9 elucidates that $m_{\mathrm{c}}$ can be expressed as a linear function of the thermal aging duration, which can be used to predict the effective modulus of CMCs after thermal aging. As the porosity of porous matrix CMCs is in the range of $30 \%-40 \%$, Fig. 10 presents the predictions of effective modulus for CMCs with a porosity of $20 \%-40 \%$.

\section{Correlation between porosity and strain energy release rate}

The phenomenological study demonstrated the importance of taking micromechanical effects into account to investigate the macroscopic failure of materials $[20,27]$. Cyclic thermal shock-induced macroscopic failure in thermal aged CMCs is mainly ruled by the nucleation phase, or initiation phase of the micro-cracks in matrix, rather than the growth and the coalescence phase that have a dominant role in the very last part of the failure process [28]. The most effective method to analyze the initiation of micro-cracks in matrix is the energy method [29], assuming that the energy is released when the critical strain energy release rate or the micro-cracks fracture toughness is reached, then the next micro-crack is formed. In the present work, the relationship between the strain energy release rate and the microstructure of thermal aged CMCs under cyclic thermal shocks is constructed by taking into account porosity.

The elastic strain energy of materials can be written as [30],

$U=\frac{1}{2} \frac{\sigma_{\mathrm{th}}^{2}}{E} V$

where $E$ is the elastic modulus of thermal aged CMCs, $\sigma_{\text {th }}$ is the thermal stress under cyclic thermal shocks and $V$ is the representative volume of interest, $V=l^{2} d$. Assuming $G$ is the strain energy release rate corresponding to the total surface area of all cracks, $A_{\text {crack }}$, then

$G=-\frac{\partial U}{\partial A_{\text {crack }}}$

here $A_{\text {crack }}=n d l, n$ is the number of cracks formed under cyclic thermal shocks, $l$ is the specimen length and $d$ is the layer thickness.

Define the crack density, $\rho$, as the ratio of the total number of matrix micro-cracks over the length of the specimens [31],

$\rho=\frac{n}{l}$

Combining Eqs. (6)-(8), $G$ is expressed as a function of crack density,

$$
-\frac{1}{2} \frac{\partial\left(\frac{\sigma_{\mathrm{th}}^{2}}{E}\right)}{\partial \rho}=G
$$

Assuming that all the matrix micro-cracks have the same volume, $V_{\text {crack }}^{i}=V_{\text {crack, }}$, the increase in matrix micro-cracks volume can be characterized by an increase in relative porosity,

$\rho s=\Delta \zeta$

where $s$ is the thickness of a single crack, and it is assumed to be a constant in this work as suggested by Refs. [27,32], $s=1.5 \mu \mathrm{m}$. Substituting Eq. (10) into Eq. (9), the following expression for $G$ can be extracted,

$-\frac{1}{2} s \frac{\partial\left(\frac{\sigma_{\mathrm{uh}}^{2}}{E}\right)}{\partial \Delta \zeta}=G$

For cyclic thermal shocks, the thermal stress $\sigma_{\text {th }}$ is proportional to the shock temperature $T_{\text {shock}}$, and can be written as [19],

$\sigma_{\mathrm{th}}=\frac{\alpha_{\mathrm{TEC}} T_{\mathrm{shock}} E}{1-\nu} \psi\left(B_{\mathrm{i}}\right)$

with a correction term $\psi\left(B_{\mathrm{i}}\right)$ depending on the convection and plate geometry. Hence, the thermal stress $\sigma_{\text {th }}$ can be obtained from the elastic modulus measured in the experiments by using Eq. (12). 


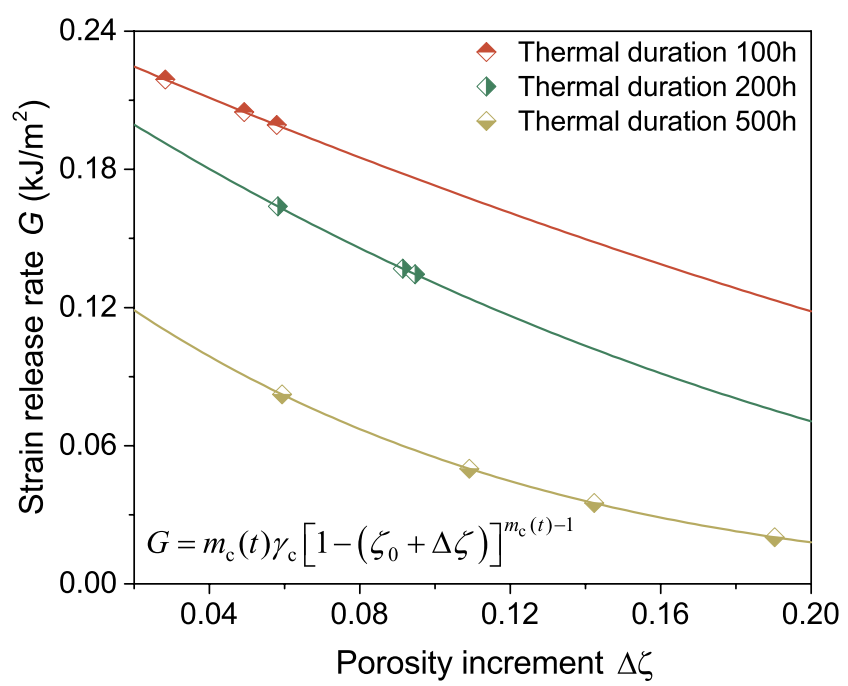

Fig. 11. Correlation between the strain energy release rate and the porosity increment $\Delta \zeta$ in the thermal aged CMCs under cyclic thermal shocks.

Substituting Eq. (5) and Eq. (12) into Eq. (11), the expression of $G$ for thermal shocks can be further simplified as [26],

$G=m_{c}(t) \gamma_{c}\left[1-\left(\zeta_{0}+\Delta \zeta\right)\right]^{m_{c}(t)-1}$

where the total porosity $\zeta=\zeta_{0}+\Delta \zeta$, and the parameter $\gamma_{c}$, affecting the strain energy release rate $G$, is defined as

$\gamma_{c}=\frac{s}{2}\left[\frac{\alpha_{\mathrm{TEC}} T_{\text {shock }}}{1-\nu} \psi\left(B_{\mathrm{i}}\right)\right]^{2}\left[\varpi \eta_{\mathrm{o}} E_{\mathrm{f}} V_{\mathrm{f}}+(1-\varpi) E_{\mathrm{m}} V_{\mathrm{m}}\right]$.

Eq. (13) shows that the relationship between the strain energy release rate and the porosity increment of thermal aged CMCs under cyclic thermal shocks is controlled by material parameter $\gamma_{c}$ and aging related parameter $m_{\mathrm{c}}(t)$. Eq. (13) can be used to describe the evolution of strain energy release rate for the thermal aged CMCs under cyclic thermal shocks.

The relationship between the strain energy release rate and the porosity increment for the thermal aged CMCs under cyclic thermal shocks is shown in Fig. 11, illustrating that the strain energy release rate $G$ is a function of the porosity increment $\Delta \zeta$ in the thermal aged CMCs under cyclic thermal shocks and it is decreased with the porosity increment.

\section{Conclusions}

In the present work, the effects of thermal aging on the cyclic thermal shock behavior of oxide/oxide CMCs were studied. The mechanical properties of the thermal aged CMCs under cyclic thermal shocks were analyzed by hierarchical porosity analysis and uniaxial compression tests. The following conclusions can be drawn:

- Two mechanical property degradation mechanisms for the thermal aged CMCs under cyclic thermal shocks are revealed in Fig. 3: (i) Delamination between the fibers and matrix within the boundary, and (ii) micro-cracks in the matrix distributed along with the thickness.

- For the compressive properties shown in Fig. 7, the thermal aged specimens showed a slight increase of elastic modulus than those of the original specimens. However, after cyclic thermal shocks, the thermal aged CMCs show a rapid decrease in elastic modulus compared to the original CMCs.

- Considering the deterioration of CMCs after thermal aging process, the aging-related factor $m_{\mathrm{c}}$ was introduced into the compress modulus prediction of the thermal aged CMCs under cyclic thermal shocks, which showed good prediction results, as illustrated in Fig. 8.

- The strain energy release rate of the thermal aged CMCs under cyclic thermal shocks shows a power-law function with the porosity increment with the thermal aging duration effects, which provides an excellent agreement with the experiments depicted in Fig. 11.

\section{Declaration of competing interest}

The authors declare that they have no known competing financial interests or personal relationships that could have appeared to influence the work reported in this paper.

\section{Acknowledgement}

The present work is financed by the China Natural Science Foundation under the contract numbers 11572169 and 51775294.

\section{References}

[1] O. Gavalda Diaz, D.A. Axinte, P. Butler-Smith, D. Novovic, On understanding the microstructure of $\mathrm{SiC} / \mathrm{SiC}$ ceramic matrix composites (CMCs) after a material removal process, Mater. Sci. Eng. A: Struct. Mater. Prop. Microstruct. Process. 743 (2019) 1-11.

[2] Y. Gowayed, J. Pierce, D. Buchanan, L. Zawada, R. John, K. Davidson, Effect of microstructural features and properties of constituents on the thermo-elastic properties of ceramic matrix composites, Compos. B Eng. 135 (2018) 155-165.

[3] Kazuya Shimoda, Tatsuya Hinoki, Yi-Hyun Park, Development of non-brittle fracture in $\mathrm{SiC}^{\mathrm{f}} / \mathrm{SiC}$ composites without a fiber/matrix interface due to the porous structure of the matrix, Compos. Appl. Sci. Manuf. 115 (2018) 397-404.

[4] Jacques Lamon, Review: creep of fibre-reinforced ceramic matrix composites, Int. Mater. Rev. (2019) 1-35.

[5] M.B. Ruggles-Wrenn, R.L. Lanser, Tension-compression fatigue of an oxide/oxide ceramic composite at elevated temperature, Mater. Sci. Eng. A: Struct. Mater. Prop. Microstruct. Process. 659 (2016) 270-277.

[6] Amir M. Parvanian, Hamid R. Salimijazi, Mohammadhossein Fathi, Mohammad Saadatfar, Synthesis and thermal shock evaluation of porous SiC ceramic foams for solar thermal applications, J. Am. Ceram. Soc. 0 (0) (2018).

[7] Z.R. Xu, K.K. Chawla, X. Li, Effect of high-temperature exposure on the tensilestrength of alumina fiber Nextel 610, Mater. Sci. Eng. A: Struct. Mater. Prop. Microstruct. Process. 171 (1-2) (1993) 249-256.

[8] Eric A.V. Carelli, Hiroki Fujita, James Y. Yang, Frank W. Zok, Effects of thermal aging on the mechanical properties of a porous-matrix ceramic composite, J. Am. Ceram. Soc. 85 (3) (2002) 595-602.

[9] V. Kostopoulos, D.E. Vlachos, A. Paipetis, G. Sotiriadis, Anisotropic damage of alumina/alumina CFCCs under long term high temperature exposure: investigations by ultrasonic stiffness measurements and quasi-static tests, Compos. Sci. Technol. 66 (16) (2006) 3221-3229.

[10] C. Cluzel, E. Baranger, P. Ladeveze, A. Mouret, Mechanical behaviour and lifetime modelling of self-healing ceramic-matrix composites subjected to thermomechanical loading in air, Compos. Appl. Sci. Manuf. 40 (8) (2009) 976-984.

[11] E. Volkmann, K. Tushtev, D. Koch, C. Wilhelmi, G. Grathwohl, K. Rezwan, Influence of fiber orientation and matrix processing on the tensile and creep performance of Nextel 610 reinforced polymer derived ceramic matrix composites, Mater. Sci. Eng. A: Struct. Mater. Prop. Microstruct. Process. 614 (Supplement C) (2014) 171-179.

[12] E. Vokmann, M.D. Barros, K. Tushtev, W.E.C. Pritzkow, D. Koch, J. Goring, C. Wilhelmi, G. Grathwohl, K. Rezwan, Influence of the matrix composition and the processing conditions on the grain size evolution of Nextel 610 fibers in ceramic matrix composites after heat treatment, Adv. Eng. Mater. 17 (5) (2015) 610-614.

[13] R.S. Hay, G.E. Fair, T. Tidball, Fiber strength after grain growth in Nextel ${ }^{\mathrm{tm}} 610$ alumina fiber, J. Am. Ceram. Soc. 98 (6) (2015) 1907-1914.

[14] Randall S. Hay, Kristin A. Keller, Larry P. Zawada, Nathan S. Jacobson, Geoff E. Fair, Degradation of Nextel ${ }^{\mathrm{tm}}$ 610-based oxide-oxide ceramic composites by aluminum oxychloride decomposition products, J. Am. Ceram. Soc. 101 (9) (2018) 4203-4223.

[15] R.S.M. Almeida, E.L. Bergmuller, H. Luhrs, M. Wendschuh, B. Clauss, K. Tushtev, K. Rezwan, Thermal exposure effects on the long-term behavior of a mullite fiber at high temperature, J. Am. Ceram. Soc. 100 (9) (2017) 4101-4109.

[16] C. Kastritseas, P.A. Smith, J.A. Yeomans, Damage characterisation of thermally shocked cross-ply ceramic composite laminates, J. Mater. Sci. 41 (3) (2006) 951-962.

[17] V. Sabelkin, G. Joshi, S. Mall, W.J. Porter, R. John, Monotonic tension and creep behavior of single crystal cmsx-486 under combustion environment, Mater. Sci. Eng. A: Struct. Mater. Prop. Microstruct. Process. 569 (2013) 106-116, 2013.

[18] K. Andreev, V. Tadaion, Q. Zhu, W. Wang, Y. Yin, T. Tonnesen, Thermal and mechanical cyclic tests and fracture mechanics parameters as indicators of thermal shock resistance-case study on silica refractories, J. Eur. Ceram. Soc. 39 (4) (2019) 1650-1659. 
[19] Zhengmao Yang, Yuan Huang, Hui Liu, Evolution and characterization of cyclic thermal shock-induced thermomechanical damage in oxide/oxide ceramics matrix composites, Int. J. Fatigue 120 (2019) 150-161.

[20] Z. Yang, Y. Huang, B. Markert, Representation of micro-structural evolution and thermo-mechanical damage in thermal shocked 2-D woven oxide/oxide ceramic matrix composites, Int. J. Fatigue 126 (2019) 122-129.

[21] B. Moser, A. Rossoll, L. Weber, O. Beffort, A. Mortensen, Nextel ${ }^{t m} 610$ alumina fibre reinforced aluminium: influence of matrix and process on flow stress, Compos. Appl. Sci. Manuf. 32 (8) (2001) 1067-1075.

[22] B. Moser, A. Rossoll, L. Weber, O. Beffort, A. Mortensen, Damage evolution of Nextel $610^{t m}$ alumina fibre reinforced aluminium, Acta Mater. 52 (3) (2004) 573-581.

[23] C. Ben Ramdane, A. Julian-Jankowiak, R. Valle, Y. Renollet, M. Parlier, E. Martin, P. Diss, Microstructure and mechanical behaviour of a Nextel ${ }^{t m} 610$ /alumina weak matrix composite subjected to tensile and compressive loadings, J. Eur. Ceram. Soc. 37 (8) (2017) 2919-2932.

[24] Donald R. Askeland, Pradeep P. Fulay, Wendelin J. Wright, The Science and Engineering of Materials, sixth ed., Wiley international, New York, 2010.

[25] Bo Madsen, Anders Thygesen, Hans Lilholt, Plant fibre composites-porosity and stiffness, Compos. Sci. Technol. 69 (7) (2009) 1057-1069.
[26] Zhengmao Yang, Hui Liu, Yuan Huang, Micro-porosity as damage indicator for characterizing cyclic thermal shock-induced anisotropic damage in oxide/oxide ceramic matrix composites, Eng. Fract. Mech. 220 (2019), 106669.

[27] C. Vinet, P. Priou, Micromechanical damage model taking loading-induced anisotropy into account, Aero. Sci. Technol. 1 (1) (1997) 65-76.

[28] G. Guillamet, A. Turon, J. Costa, J. Renart, P. Linde, J.A. Mayugo, Damage occurrence at edges of non-crimp-fabric thin-ply laminates under off-axis uniaxial loading, Compos. Sci. Technol. 98 (2014) 44-50.

[29] Siulie Liu, John A. Nairn, The formation and propagation of matrix microcracks in cross-ply laminates during static loading, J. Reinf. Plast. Compos. 11 (2) (2016) $158-178$.

[30] V. Vinogradov, Z. Hashin, Probabilistic energy based model for prediction of transverse cracking in cross-ply laminates, Int. J. Solids Struct. 42 (2) (2005) 365-392.

[31] D.T.G. Katerelos, J. Varna, C. Galiotis, Energy criterion for modelling damage evolution in cross-ply composite laminates, Compos. Sci. Technol. 68 (12) (2008) 2318-2324.

[32] L.R. Dharani, L. Chai, N.J. Pagano, Steady-state cracking in ceramic matrix composites, Compos. Sci. Technol. 39 (1) (1990) 29-43. 